\title{
An IoT-based Mobile Gateway for Intelligent Personal Assistants on Mobile Health Environments
}

\author{
João Santos ${ }^{1}$, Joel J. P. C. Rodrigues ${ }^{1,2,3}$, Bruno M. C. Silva ${ }^{1}$, João Casal $^{4}$, Kashif Saleem ${ }^{3}$, Victor Denisov ${ }^{5}$ \\ ${ }^{1}$ Instituto de Telecomunicações, University of Beira Interior, Covilhã, Portugal \\ ${ }^{2}$ University of Fortaleza (UNIFOR), Ceará, Brazil \\ ${ }^{3}$ Center of Excellence in Information Assurance (CoEIA), King Saud University (KSU), Riyadh, Saudi Arabia \\ ${ }^{4}$ TIMWE I \&D, Parkurbis - Parque de Ciência e Tecnologia da Covilhã, Portugal \\ ${ }^{5}$ ITMO University, St. Petersburg, Russia
}

joao.santos@it.ubi.pt; joeljr@ieee.org; bruno.silva@it.ubi.pt; joao.casal@timwe.com; ksaleem@ksu.edu.sa; 070255@gmail.com

\begin{abstract}
The evolution of mobile devices has triggered the appearance of intelligent personal assistants (IPAs). IPAs are software agents used to support users to fulfill several daily actions. They are supposed to be intelligent in such a way that allows them to give their owners advices about many different subjects. To do so, IPAs must learn about their user behavior and routines. With the current state of the art technologies, scenarios of ubiquitous communication can be created. One of the potential enablers for those scenarios is the Internet of Things (IoT) paradigm where machines with decision support systems interact and communicate among them. In an IoT environment, IPAs can interact with other smart objects in order to gain new knowledge and awareness about their users. This paper proposes a novel IoT-based mobile gateway solution for mobile health (m-Health) scenarios. This gateway autonomously collects information about the user/patient location, heart rate, and possible fall detection. Moreover, it forwards the collected information to a caretaker IPA, in real time, that will manage a set of actions and alarms appropriately. The algorithms used for each mobile gateway service, and the scenarios where the mobile gateway acts as a communication channel or a smart object are also addressed on this paper.
\end{abstract}

Keywords- Intelligent Personal Assistant; Mobile Health; Body Sensor Network; Internet of Things; Mobile Gateway; Smart Object

\section{Introduction}

The use of personal assistants is not a new trend, since they are used through daily business and personal tasks for many years. They are responsible for managing several daily routines, such as paying bills, making appointments, shopping, taking notes, dealing with correspondence, answering phone calls, among others. Intelligent personal assistants (IPAs) are software agents that can automate and ease many of the daily tasks of their users [1][2]. IPAs gather knowledge and awareness about the usual behavior of their users by interacting with them and/or collecting awareness data (e.g., location and context). Therefore, IPAs are supposed to adapt themselves to their user's needs and actions, in order to improve the given assistance.

The appearance of the Internet of Things (IoT) paradigm enables the creation of ubiquitous communication scenarios, where all addressable objects in the environment are supposed to communicate and cooperate [3][4][5]. IoT offers new technological opportunities to create new types of services that users can benefit for both their personal and professional lives [6]. Services where humans access other remote machines to perform some desired tasks will be available. Smart Homes [7][8], Smart Cities [9], manufacturing [10], healthcare [11][12], transports and logistics [13][14], and smart grids [15] are some examples of areas on which IoT is already creating new business opportunities.

The inclusion of IPAs on IoT scenarios can enhance those devices capabilities to gain more knowledge and awareness about their surroundings. IPAs can even better perceive the preferences of their users by learning through their interactions with other smart objects. For example, an IPA could learn the morning routine of its user by interacting with a smart object in the environment, such as a toaster, an air conditioner, or a coffee machine. Gateways are often used to establish a connection between the IoT environment and the Internet. Static gateways are used on static environments while mobile gateways can be useful on dynamic environments with high user mobility.

The use of smart mobile devices offers numerous opportunities to create efficient healthcare services and solutions. Mobile health (m-Health) applications use mobile devices to deliver healthcare services anytime and anywhere, surpassing geographical, temporal, and even organizational barriers. M-Health solutions address emerging problems such as the increasing number of chronic diseases related to lifestyle, high costs of existing 
national health services, and the need to empower patients and families to self-care [16].

This paper presents a novel mobile gateway solution for ubiquitous $\mathrm{m}$-Health scenarios where a body sensor network (BSN) [17] monitors a person in real-time and collects information about his/her environment. The BSN collects information about the location and the heart rate of the user, and also detects if he/she has fallen. Each one of these items is mapped to an individual service on the mobile gateway application deployed in a smartphone. The gateway is responsible for sending the information of each service to a caretaker IPA platform called AMBRO. There, the caretaker receives notifications and alerts regarding the person being monitored. This paper also presents a prototype to demonstrate and validate the proposed solution. The evaluation of the mobile gateway is performed through the assessment of its power consumption, the accuracy of its services, and the way that it communicates with other objects on the environment. The main contributions of this paper are the following:

- A novel IoT-based mobile gateway solution integrated with an IPA platform;

- An IPA platform (AMBRO) for multiple environments (smart home and healthcare);

- A novel remote monitoring system for a ubiquitous m-health scenario.

The remainder of the paper is organized as follows. Section 2 presents a literature review about IPAs, remote monitoring systems, and IoT gateways. Section 3 briefly describes the AMBRO platform, including its layered architecture, while the AMBRO mobile gateway and its different services are addressed in Section 4. The experiments performed to evaluate the AMBRO mobile application and the analysis of the results are considered in Section 5, while Section 6 presents the conclusions, suggesting further research work.

\section{Related Work}

IPAs can be integrated on healthcare environments as tools to assist patients, physicians or caretakers. For instance, an IPA can be used as a medication reminder, and give real time information about the health status of monitored patients to the medical staff. The available mobile technology on smartphones enabled the creation of well-known personal digital assistants as Apple's Siri [18], Google Now [19], Samsung's S Voice [20], and Microsoft's Cortana [21].

In recent years, some IPAs for the healthcare context have been developed. The work presented in [22] demonstrates an example of an Android application, called SapoMed, used to manage the medication time schedule of the users. It allows users to register different drugs to the medication intake schedule, and uses a Web service to present the prescription information about each registered medication. SapoMed application can be seen as a mobile personal assistant since it generates alerts to remind their users about the time they have to take the medication. SapoFit, presented in [23], is a weight control mobile application for obesity prevention. This application allows users to manage and keep track of weight in a healthier and practical way. Based on the previous user input information, SapoFit offers several features, such as the weight control, body mass index (BMI) and basal metabolic rate (BMR) calculation, sports activity monitoring, and healthy meal planning. In [24] the authors proposed a health monitoring system, denominated HealthPal, that interacts with peripheral devices (such as thermometers) and personal digital assistants (PDAs) to create an insightful record of the patients' health status. The system used by elderly people considers an application installed on their PDAs and on the medical staff devices (computers, PDAs, etc.) to receive alerts if some health status indicator is alarming. The solution presented in [25] notifies autonomously a caretaker when a fall is detected for its user. In this scenario, the mobile device used by the caretaker only needs to receive the information gathered by the BSN (when a fall is detected) to display it to the caretaker. In [26], a platform used to monitor the health status of persons at their home environment is presented. The system comprises a (i) wireless sensor network (WSN) to monitor the house environment, (ii) an online platform to store the data gathered from sensors, and (iii) end-user devices, such as computers, mobile phones and tablets, to be used by the medical staff or other informal caretakers. The online platform is able to detect abnormal values on the health indicators of the monitored person and then generate notifications to the caretakers' devices. A study about the use of WSNs on health monitoring scenarios is presented in [27]. Body sensors (blood pressure, electrocardiogram, heart rate, among others) and environmental sensors are used to estimate the health status of persons being monitored. Those two types of networks are connected to a backbone where the data gathered by the sensors is stored in a central database. End-user devices, such as PDAs, are also connected to the backbone in order to access to the database. In [28], the CodeBlue project is presented. It describes a platform where caregivers can subscribe to physiological parameters that are gathered by sensors monitoring persons under their care. The caregivers can access the health status information of the monitored persons on their computers or PDAs. Other interesting m-health network architectures based on WSNs are presented in [29], with the WSN4QoL project, and [30], with the description of several research projects for m-Health applications. Works presenting novel solutions to solve some issues on BSNs used on healthcare applications, such as the energy efficiency, and the data reliability, are also presented on [31] and [32]. 
On m-Health solutions, it can be necessary to forward the traffic originated by sensors to external networks (such as the Internet). Then, device gateways can be used. An architecture for IoT scenarios is presented in [33]. It comprises a semantic gateway that is able to understand different communication protocols, such as the constrained application protocol (CoAP), the extensible messaging and presence protocol (XMPP), and the message queue telemetry transport (MQTT). This work shows an interesting study about the mechanisms that are necessary to develop an interoperable gateway in different environments. There are also presented some services used at the gateway that differentiate the incoming traffic, which eases the creation of meaningful information. However, there is not detailed how this semantic gateway can be used in a real deployment, including its capability to work on dynamic environments. The work presented in [34] shows a Power over Ethernet (PoE) gateway used at a medical environment, where sensors are used to check the health status of the patients. This stationary gateway is deployed on a platform similar to the Arduino where an Ethernet connection is used to power the gateway. The gateway is able to communicate with healthcare sensors over Wi-Fi, Bluetooth or IEEE 802.15.4 connections. The usage of this gateway is not possible on scenarios with mobility demands, since it always needs to be powered up via an Ethernet connection to operate correctly. In [35], the authors presented a ubiquitous personal health surveillance and monitoring system composed by a BSN and a gateway. The gateway's role is performed by an Android smartphone, which receives the data gathered by the BSN and forwards it to the health database servers on the Internet. Then, the medical staff is able to properly analyze the health indicators of their patients. The gateway communicates with the BSN through a sink node using Bluetooth or USB connections. However, little information is provided about the data throughput and the power consumption of the network devices.

When compared with other solutions presented in the literature, the AMBRO mobile gateway proposed in this paper differs from them because it has the ability to communicate with sensors while, at the same time, it decides how the communication process is managed. Also, it offers the possibility to control the power consumption on the system devices (as the smartphone), while the monitoring services accuracy is preserved, making the AMBRO mobile gateway an application capable to use accurate and low-power health monitoring services. Moreover, the AMBRO mobile gateway is supposed to be used on scenarios of high mobility, creating ubiquitous communication scenarios where persons can be constantly monitored. Next sections will present this solution in detail.

\section{The AMBRO Platform}

AMBRO is a technological platform [36] that supports the integration of IPAs on ubiquitous communication environments. AMBRO was developed to assist his/her user on numerous ways, giving suggestions about several aspects of the daily life. Using an AMBRO personal assistant, the users can (i) access Web services of their interest (e.g., find nearer restaurants, consult the weather information, etc.), (ii) consult the information about their body sensors under use (see the temperature for instance), and (iii) change the behavior of smart objects in the environment (turn off the air conditioner, among others). Besides these aspects, the AMBRO personal assistant is capable to learn the usual behavior of their users by interpreting their interactions with other objects in the environment, including the personal assistant itself.

The AMBRO platform considers three logical layers, i) the IoT world, ii) the intelligent cloud system, and iii) the user interfaces. The IoT layer includes all the external devices (objects and sensors) that gives IPAs the information about the environment that surrounds them. The static gateway available on this layer is responsible for managing the communication between sensors and objects in the environment, and the cloud. The static gateway supports different communication protocols, such as the ZigBee and the Z-Wave. An AMBRO proprietary protocol is also supported. As for the mobile gateway, which is one of the main contributions of this paper, it interconnects the BSN to the AMBRO platform. It was developed for the Android operating system, and connects with the AMBRO cloud using RESTful Web services. The sensors on the IoT world layer are used to retrieve information on the user environment. The periodicity chosen to collect data about the environment variables is carried out independently by each sensor, allowing the user to adjust it as he/she wants. Objects, such as fans or light bulbs, can be operated automatically or operated manually by the user.

The intelligent cloud system layer is the core of the AMBRO platform, being responsible for all the logical actions concerning IPAs. It includes the usage of databases for data storage; a "security and authentication" module to assure the integrity of the information flowing on the system; an "artificial intelligence (AI)" module to allow learning mechanisms to be used on data gathered by the system; a "text-to-speech module" to synthesize text strings and files into voice; a "voice recognition" module to convert spoken audio into text strings; a "notifications" module to allow IPAs to receive notifications about events happening on the system; and a "communication" module to manage the interaction between the IPAs and other external entities.

At the user interfaces layer, the interfaces to allow the users to interact with the AMBRO system are provided. One of them is the back-office tool that is used to manage the user profile, sensors, and others objects. The other interfaces correspond to the personal assistant interfaces where can be emphasized the IPA Web, which is 
accessible in every device with Internet connectivity; the IPA mobile that can be used on Android (minimum version 4.0.3) and $\mathrm{iOS}$ (minimum version 8.1) smartphones; and the IPA smart watch, available on any Android Wear OS smart watch. The IPA smart watch is mostly used as a complement to the mobile IPA interface. The smart watch should be paired with an Android smartphone, and mostly displays system notifications regarding sensors and objects. Figure 1 depicts the AMBRO platform architecture.

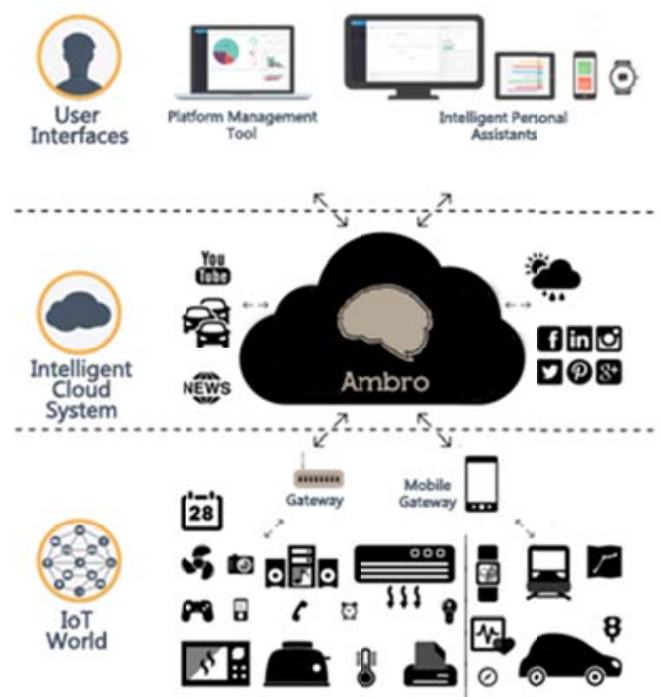

Fig. 1. AMBRO platform main architecture.

\section{AMBRO Mobile Gateway}

On communication scenarios where devices are constantly changing their position, it can be necessary to use a mobile gateway to forward the traffic. The use of a smartphone as a mobile gateway is a possible solution to enable such scenarios. This paper presents a solution exploited through a mobile application (developed for Android operating system), identified to as AMBRO mobile gateway. The AMBRO mobile gateway is a specific gateway application that is placed on a remote health-monitoring scenario, allowing a person that possesses an AMBRO personal assistant (the caretaker) to check some health indicators about a patient under his/her care. In order to make the system fully functional, the monitored person must always have with him a smartphone with the AMBRO mobile gateway application installed. This application allows both actors (monitored person and caretaker) to control three different monitoring services that use BSN sensors. The user should authenticate on the application using his/her AMBRO account credentials. Those credentials are the same that are used by the caretaker on his AMBRO personal assistant since, in practice, the mobile gateway belongs to him/her. Figure 2 shows the splash screen of the AMBRO mobile gateway application.

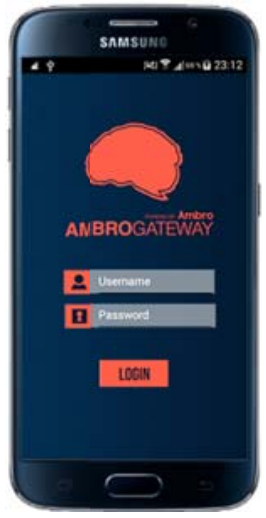

Fig. 2. AMBRO mobile gateway splash screen.

After correct authentication, the user goes to the AMBRO mobile gateway main screen (shown in Figure 3) where he/she has the possibility to start or stop the monitoring services. Besides that, the user is also able to see relevant information about the data gathered by each monitoring service, such as the coordinates of his/her current location, his/her heart beats per minute, and the number of times he/she potentially fell. One other service available on the mobile gateway application is related with the search of Bluetooth objects to be used on the monitoring services.
Fig. 3. Main screen of the AMBRO mobile gateway.

The AMBRO mobile gateway architecture considers four modules, as depicted in Figure 4: i) the BSN, ii) the mobile gateway, iii) the AMBRO cloud, and iv) the IPA of the caretaker. The BSN module is composed by sensors that monitor the person in real time. On this case, there are two types of devices: one Shimmer [37] using an accelerometer and a gyroscope for fall detection, and a smart watch with an embedded heart rate sensor to count the number of heart beats. The information gathered by both sensors is transferred to the mobile gateway through a Bluetooth connection (the smart watch uses Bluetooth Low Energy, while the Shimmer module uses Bluetooth class 2). The mobile gateway module corresponds to the 
smartphone that has the AMBRO mobile gateway installed, which allow the monitoring services to be controlled. One of those monitoring services (the location monitoring service) uses the GPS receiver of the smartphone to accurately determinate the position of the monitored person. The data gathered by the monitoring services is forwarded to the AMBRO cloud through RESTful Web services by using the HTTP. Inside the AMBRO cloud, the information is stored and then processed by the multiple logical modules available there. If some indicator about the monitored person is alarming, a notification is generated to the caretaker IPA through a push notification mechanism.

\subsection{Location monitoring service}

The location monitoring service is a tracking service used to retrieve the position of the user through the smartphone GPS. Using the low power embedded sensors of the smartphone (e.g., barometer, gyroscope, accelerometer), this service is also able to discover the activity performed by the user. This is possible by using the Google's Activity Recognition API [38], which enables the detection of multiple possible activities for the user (e.g., still, walking, running, driving a vehicle, riding a bicycle).

Other works have been published concerning tracking systems like the one being presented at the AMBRO mobile gateway. In [39], the authors presented a location based application for Android, capable of changing the profile on the smartphone according to the user location; locating a person through a SMS; locating people on nearby places to the user. In [40] a vehicle tracking system using the GPS system of an Android smartphone is proposed. The application can be useful for monitoring the driver performance, check the speed at a vehicle circulates, or increase the performance of fleet management businesses. The authors of [41] proposed a smartphone tracking system used to retrieve children's current location. The application is designed to help parents tracking their children in real time, by sending SMS messages to the smartphones of their children. The children's smartphones answer with their current location signaled on a map. In [42], a mobile tracking application for Android using GPS is proposed. The application tracks children through their smartphones, defining geographical areas that cannot be surpassed. The parents are warned through a SMS message if their child is outside the allowed geographical area.

Regarding the location monitoring service of the AMBRO mobile gateway, it can only be activated if the Wi-Fi and GPS have been previously enabled on the smartphone. When this is assured, the location monitoring service is initiated and activity updates are requested every 20 seconds. If the low power embedded sensors of the smartphone detect the user is moving, GPS is turned on, and location updates are requested. Location updates are stopped when the smartphone detects the user is stopped during one straight minute. This mechanism is important to diminish the power consumption on the smartphone, since the GPS is only active when the user is moving.

The location monitoring service allows data (coordinates and activity detected) to be stored on the smartphone memory if the Internet connection fails. The smartphone retries to send the data to the AMBRO cloud every time an activity detection update is requested, assuring that no location updates are lost. If the coordinates sent by the mobile gateway to the AMBRO cloud return a place outside a geo-reference defined dynamically by the system, the caretaker is notified by an alert message on his/her IPA.

\subsection{Heart rate monitoring service}

The heart rate monitoring service is used to check its user heart rate. Then, an optical heart rate sensor embedded on an Android Wear smart watch is used. The use of an Android Wear smart watch (in this case, a Moto 360) eased the development of the service, since applications built on the Android Wear OS are very similar to the Android OS. Besides, the use of an Android Wear wearable device facilitated the communication between the smartphone and the smart watch, eliminating a problem inherent to the IoT networks: the communication between heterogeneous devices [43].

To start the service, the Bluetooth needs to be enabled on both the smartphone and the smart watch. The Wi-Fi also needs to be enabled on the smartphone. If this is all assured, the service is started with the smartphone trying to establish a Bluetooth Low Energy (BLE) connection with the smart watch. When the connection is established, the smartphone starts a mechanism to detect the activity the user is performing every 20 seconds using the Google's Activity Recognition API. This mechanism is important to detect if the user heart rate values are normal according to his/her performed activity. On the smart watch the user heart rate is detected through the optical heart rate sensor at every second. This information is sent to the smartphone through the BLE connection previously established, using the Wearable Message API [44]. Using this API, it was possible to create a wearable listener on the smartphone to make it aware of every message sent by the smart watch. The Wearable Message API mechanism is depicted in Figure 5. 


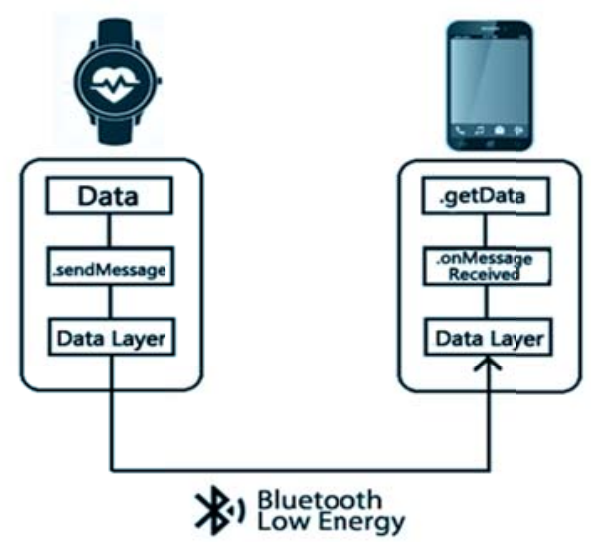

Fig.5. Wearable Message API used on the communication between the smart watch and the smartphone on the Heart rate monitoring service.

The heart rate values sent to the smartphone (mobile gateway) are summed up, and a mean of the user heart rate on the past five minutes is calculated. The gateway tries to send information to the AMBRO cloud every five minutes, containing the mean of the heartbeat and the activity the user performed along that period.

On the AMBRO cloud there are two different typical mean values for the user: user stopped and user in movement. Those values are obtained through the use of AI algorithms. If the heartbeat values sent to the AMBRO platform are too low or too high when compared with the typical heartbeat mean values of the user for that activity, a notification is sent to the caretaker. Therefore, the caretaker is informed of possible health problems on the person under care by evaluating odd heartbeat values detected by the heart rate monitoring service. If the data is not successfully delivered to the cloud, the mobile gateway stores the information locally to forward later. This is performed every 5 minutes.

\subsection{Fall detection service}

The fall detection service is used to detect a user fall. To detect a fall, one of the available possibilities is the use of tri-axis accelerometers. This mechanism allows an accelerometer attached to the body of a person to measure the acceleration of the person relatively to the gravity, using the equation (1).

$$
|A|=\frac{\sqrt{\left(A_{x}\right)^{2}+\left(A_{y}\right)^{2}+\left(A_{z}\right)^{2}}}{9.8}
$$

The use of gyroscopes can improve the fall detection systems precision. Gyroscopes can detect the orientation of a person by calculating his angular velocity through the equation (2).

$$
|\omega|=\sqrt{\left(\omega_{x}\right)^{2}+\left(\omega_{y}\right)^{2}+\left(\omega_{z}\right)^{2}}
$$

On both situations, the values are compared with thresholds pre-defined by the user, or dynamically defined by the system. There is some work published that use this methodology to detect falls. On the work presented in [45] a fall detection system is tested using a tri-axial accelerometer and a tri-axis gyroscope. By collecting data through some experiments in multiple daily life activities, the authors defined upper and lower thresholds for both acceleration and angular velocity. Then, using these thresholds, falls could be detected through acceleration and angular velocity values measured in real-time. In [46], the authors used the 3-axis accelerometer and magnetometer of a smartphone to detect falls. Again, thresholds were defined for both accelerometer and magnetometer data, defining an algorithm able to detect falls when certain values below lower thresholds and values above upper thresholds were obtained.

To develop the fall detection system on the AMBRO mobile gateway, there were done some previous experiments with a Shimmer module (containing an accelerometer and a gyroscope) to assess the typical values for the acceleration and angular velocity when the user was performing different daily life activities. These values are shown on Table I. This allowed the definition of appropriated acceleration and angular velocity thresholds.

Table I. Acceleration and angular velocity values for different daily life activities.

\begin{tabular}{|c|c|c|}
\hline \multirow{2}{*}{$\begin{array}{c}\text { Activity } \\
\text { Performed }\end{array}$} & \multicolumn{2}{|c|}{ Physical Measures } \\
\cline { 2 - 3 } & $\begin{array}{l}\text { Acceleration (estimate) } \\
{[G]}\end{array}$ & $\begin{array}{l}\text { Angular Velocity (estimate) } \\
\text { [degrees/second] }\end{array}$ \\
\hline Still & $0.9-1.15$ & $0-90$ \\
\hline Fall & $0.05-3.5$ & $150-300$ \\
\hline Walk & $0.4-1.7$ & $0-100$ \\
\hline Sit Down & $0.3-2.4$ & $30-140$ \\
\hline Stand Up & $0.6-1.4$ & $30-140$ \\
\hline Lay Down & $0.5-1.5$ & $80-200$ \\
\hline Run & $0.2-2.9$ & $15-170$ \\
\hline Jump & $0.4-3$ & $80-170$ \\
\hline
\end{tabular}

As shown in Table I, when a fall is detected the acceleration can change from $0.05 \mathrm{G}$ to $3.5 \mathrm{G}$. The lower acceleration value is detected when a person is at free fall. The higher acceleration value is usually correspondent to the instant the person hits the floor. The correct combination of the Lower Acceleration Threshold (LAT), the Upper Acceleration Threshold (UAT), and the 
Angular Velocity Threshold (AVT) can create a trustworthy mechanism to detect a fall. To do so, there were defined three reasonable values for each threshold: $0.3 \mathrm{G}$ to the LAT, $2.5 \mathrm{G}$ to the UAT, and $220^{\circ}$ to the AVT. The definition of these thresholds tried to optimize the fall detection mechanism, avoiding the false positives, and avoiding misunderstanding between a fall and other daily life activities, especially a run or a jump.

To activate the fall detection service at the AMBRO mobile gateway, the user should primarily choose the Shimmer sensor that he/she wants. After that, the mobile gateway establishes a Bluetooth connection with the Shimmer sensor module that was chosen. The Shimmer module starts streaming every 20 milliseconds, gathering values from the accelerometer and from the gyroscope. A fall is detected every time the UAT is exceeded and, in the next 0.8 seconds, at least three values below the LAT are detected. If this premise is fulfilled, it is possible that a person is at free fall. To confirm that a person is effectively falling, it is needed to ascertain if in the next 1.5 seconds, the acceleration surpasses again the UAT, and the angular velocity exceeds the AVT. If all this is true, one last step needs to be performed. The mean value for the acceleration in the next two seconds needs to be less than $1.2 \mathrm{G}$, indicating the person is still, and possibly lying on the floor. This indicates that a fall has been detected. The AMBRO mobile gateway sends a notification to the caretaker IPA to inform about the situation. If the notification fails to be delivered, the mobile gateway will try to resend it one minute later. This process will repeat until the notification has been delivered or the fall detection service has been stopped by the user.

\subsection{Object search service}

The object search service is used to discover sensors that communicate over Bluetooth, like the Shimmer devices. The system will enable the Bluetooth adapter automatically on the smartphone if it is not enabled when the service tries to start.

Firstly, the gateway sends a broadcast to search Bluetooth devices. When the discovery process is over, a list of Bluetooth devices that answered positively the broadcast message is returned to the user. Then, the user can select one of those devices to be paired with the gateway. If the device that has been paired with the gateway is a Shimmer sensor, it will be eligible to be used on the fall detection service. Figure 6 illustrates this process showing a list of devices returned by the object search service on the AMBRO mobile gateway.

The object search service can only be launched if the heart rate monitoring service and the fall detection service are not running. This mechanism prevents the degradation of the communication between the mobile gateway and other Bluetooth devices, since it does not allow the communication environment to be burdened by Bluetooth broadcast messages.

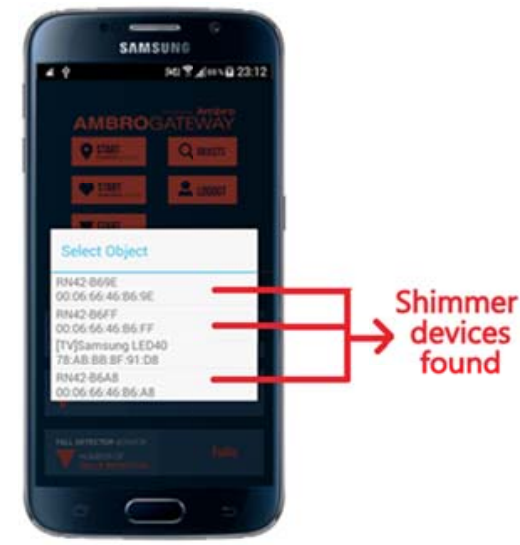

Fig.6. Illustration of the object search service usage, showing a list of devices returned to the AMBRO mobile gateway.

\section{Results Analysis}

There were defined three metrics to assess the performance of the AMBRO mobile gateway: $i$ ) power consumption of the devices; ii) accuracy of each monitoring service available at the mobile gateway; and iii) the interoperability with other objects available on the environment.

\subsection{Power Consumption}

The devices' power consumption in IoT environments is very important to evaluate the system performance on such scenarios [47]. This is due to the characteristics presented by IoT devices, such as high mobility, limited power supply, and capacity to communicate anytime and anywhere. To respect all these conditions without degrading the system performance, some strategies need to be applied. One of those strategies considers that objects only communicate when it is strictly necessary. By doing this, the quick discharge of the devices' batteries is avoided. Different experiments were conducted on the AMBRO mobile gateway application to evaluate the parameters that were changeable to make the power consumption of the system optimized. For the location monitoring and heart rate monitoring services, different experiments were performed by simply changing the rate on which activity detection updates were requested. Another parameter changed for each experiment performed on the heart rate monitoring service was the periodicity at the data was sent to the AMBRO cloud. On the fall detection service, the reading frequency for the acceleration and for the angular velocity on the Shimmer module changed for each scenario the AMBRO mobile gateway was experimented. To perform the experiments for the mobile gateway role, it was used a Samsung Galaxy Express 2, equipped with the Android OS 4.4.2, CPU dual-core $1.7 \mathrm{GHz}$, internal memory up to 
8 GB, 1.5 GB RAM, BLE connectivity, and Wi-Fi, 2G, $3 \mathrm{G}, 4 \mathrm{G}$ and LTE wireless network connectivity. It was also used a Moto 360 smart watch, equipped with the Android Wear OS (version 5.0.2), a Texas Instruments OMAP 3 processor, 4 GB of internal memory and 512 MB RAM to measure the heart rate of a monitored person. In order to evaluate the results returned for each experimental scenario correctly, it was needed to verify how many hours the battery of the smartphone and the battery of the smart watch could handle when performing normal daily life tasks,. So, it was ascertained that both smartphone and smart watch could handle, in average, 40 hours without discharging completely their batteries.

Table II presents the first experimental scenario that evaluated the power consumption of the devices available in the AMBRO mobile gateway communication environment.

Table II. Results of the AMBRO mobile gateway for the first experimental scenario.

\begin{tabular}{|c|c|c|c|c|c|c|}
\hline \multirow{2}{*}{ Services } & \multicolumn{6}{|c|}{ Experimented Features } \\
\hline & $\begin{array}{l}\text { Activ } \\
\text { ity } \\
\text { Detec } \\
\text { tion } \\
\end{array}$ & $\begin{array}{l}\text { Chec } \\
\text { k HR }\end{array}$ & $\begin{array}{l}\text { Shim } \\
\text { mer } \\
\text { perio } \\
\text { dicity } \\
\end{array}$ & $\begin{array}{l}\text { Send } \\
\text { Data to } \\
\text { the Cloud }\end{array}$ & $\begin{array}{l}\text { Smart } \\
\text { watch } \\
\text { Battery }\end{array}$ & $\begin{array}{l}\text { Smartp } \\
\text { hone } \\
\text { battery }\end{array}$ \\
\hline $\begin{array}{l}\text { Location } \\
\text { Service }\end{array}$ & $\begin{array}{l}20 \\
\text { sec }\end{array}$ & ------ & $\begin{array}{l}---- \\
\end{array}$ & $\begin{array}{l}\text { When a } \\
\text { location } \\
\text { update is } \\
\text { requested }\end{array}$ & \multirow{3}{*}{$\begin{array}{l}11 \mathrm{~h} \\
15 \mathrm{mins}\end{array}$} & \multirow{3}{*}{$19 \mathrm{~h}$} \\
\hline $\begin{array}{l}\text { HR } \\
\text { Service }\end{array}$ & $\begin{array}{l}20 \\
\mathrm{sec}\end{array}$ & $1 \mathrm{sec}$ & |--- & 5 minutes & & \\
\hline $\begin{array}{l}\text { Fall } \\
\text { Detection } \\
\text { Service }\end{array}$ & ---- & ---- & $\begin{array}{l}20 \\
\text { msec }\end{array}$ & $\begin{array}{l}\text { When a } \\
\text { fall is } \\
\text { detected }\end{array}$ & & \\
\hline
\end{tabular}

On the first experimental scenario, the activity of the user on the location monitoring service and the heart rate monitoring service was checked at a periodicity of 20 seconds. The heart rate of the monitored person was measured every second and the collected data was sent to the AMBRO cloud every 5 minutes. The Shimmer module operated at 20 milliseconds rate for fall detection. On the location monitoring service, the communication with the AMBRO cloud was not performed periodically, since location updates were not requested if the user was not under movement. The fall detection service only generated notifications when a fall was detected, and only on those situations the communication with the cloud was performed. On this scenario, it was concluded that the smart watch battery lasted 11 hours and 15 minutes, and the smartphone battery lasted 19 hours, approximately. Table III summarizes the results of the second experimental scenario to evaluate the power consumption of the AMBRO mobile gateway application.
Table III. Results of the AMBRO mobile gateway for the second experimental scenario.

\begin{tabular}{|c|c|c|c|c|c|c|}
\hline \multirow{2}{*}{ Services } & \multicolumn{6}{|c|}{ Experimented Features } \\
\hline & $\begin{array}{l}\text { Activ } \\
\text { ity } \\
\text { Detec } \\
\text { tion }\end{array}$ & $\begin{array}{l}\text { Chec } \\
\text { k HR }\end{array}$ & $\begin{array}{l}\text { Shim } \\
\text { mer } \\
\text { perio } \\
\text { dicity }\end{array}$ & $\begin{array}{l}\text { Send } \\
\text { Data to } \\
\text { the Cloud }\end{array}$ & $\begin{array}{l}\text { Smart } \\
\text { watch } \\
\text { Battery }\end{array}$ & $\begin{array}{l}\text { Smartp } \\
\text { hone } \\
\text { battery }\end{array}$ \\
\hline $\begin{array}{l}\text { Location } \\
\text { Service }\end{array}$ & $\begin{array}{l}40 \\
\text { sec }\end{array}$ & ----- & ----- & $\begin{array}{l}\text { When a } \\
\text { location } \\
\text { update is } \\
\text { requested }\end{array}$ & \multirow{3}{*}{$\begin{array}{l}11 \mathrm{~h} \\
15 \mathrm{mins}\end{array}$} & \multirow{3}{*}{$\begin{array}{l}19 \mathrm{~h} \\
15 \mathrm{mins}\end{array}$} \\
\hline $\begin{array}{l}\text { HR } \\
\text { Service }\end{array}$ & $\begin{array}{l}40 \\
\mathrm{sec}\end{array}$ & $1 \mathrm{sec}$ & ----- & 5 minutes & & \\
\hline $\begin{array}{l}\text { Fall } \\
\text { Detection } \\
\text { Service }\end{array}$ & ---- & ---- & $\begin{array}{l}50 \\
\mathrm{msec}\end{array}$ & $\begin{array}{l}\text { When a } \\
\text { fall is } \\
\text { detected }\end{array}$ & & \\
\hline
\end{tabular}

Changes can be noticed on some parameters when comparing the first experimental scenario with the second. Essentially, the activity detection rate on the second scenario was twice when compared with the first experimental scenario. Doing this, the smartphone battery could be a little spared. On the fall detection service, the Shimmer module read the acceleration and the angular velocity at a 50 milliseconds rate. The heart rate of the user was checked every second as in the first scenario. Then, the smart watch battery lifetime on the both scenarios was the same. On this second scenario the battery of the smartphone lasted 19 hours and 15 minutes, approximately. Table IV shows the results of the last experimental scenario performed on the AMBRO mobile gateway application to evaluate the power consumption on the devices.

Table IV. Results of the AMBRO mobile gateway for the third experimental scenario.

\begin{tabular}{|c|c|c|c|c|c|c|}
\hline \multirow{2}{*}{ Services } & \multicolumn{6}{|c|}{ Experimented Features } \\
\hline & $\begin{array}{l}\text { Activ } \\
\text { ity } \\
\text { Detec } \\
\text { tion } \\
\end{array}$ & $\begin{array}{l}\text { Chec } \\
\text { k HR }\end{array}$ & $\begin{array}{l}\text { Shim } \\
\text { mer } \\
\text { perio } \\
\text { dicity }\end{array}$ & $\begin{array}{l}\text { Send } \\
\text { Data to } \\
\text { the Cloud }\end{array}$ & $\begin{array}{l}\text { Smart } \\
\text { watch } \\
\text { Battery }\end{array}$ & $\begin{array}{l}\text { Smartp } \\
\text { hone } \\
\text { battery }\end{array}$ \\
\hline $\begin{array}{l}\text { Location } \\
\text { Service }\end{array}$ & $\begin{array}{l}60 \\
\mathrm{sec}\end{array}$ & ------ & ----- & $\begin{array}{l}\text { When a } \\
\text { location } \\
\text { update is } \\
\text { requested }\end{array}$ & \multirow{3}{*}{$\begin{array}{l}11 \mathrm{~h} \\
15 \mathrm{mins}\end{array}$} & \multirow{3}{*}{$\begin{array}{l}19 \mathrm{~h} \\
30 \mathrm{mins}\end{array}$} \\
\hline $\begin{array}{l}\text { HR } \\
\text { Service }\end{array}$ & $\begin{array}{l}60 \\
\text { sec }\end{array}$ & $1 \mathrm{sec}$ & ----- & 5 minutes & & \\
\hline $\begin{array}{l}\text { Fall } \\
\text { Detection } \\
\text { Service }\end{array}$ & --- & --- & $\begin{array}{l}100 \\
\text { msec }\end{array}$ & $\begin{array}{l}\text { When a } \\
\text { fall is } \\
\text { detected }\end{array}$ & & \\
\hline
\end{tabular}

On the third experimental scenario, the activity detection was performed every minute. The Shimmer module analysed the acceleration and the angular velocity every 100 milliseconds. The information relative to the heart rate monitoring service was delivered to the cloud every 10 minutes. These changes allowed the smartphone 
battery to last 19 hours and 30 minutes (more 30 minutes than the first experimental scenario, and more 15 minutes than the second experimental scenario).

Although the variation of the parameters on the third experimented scenario allowed the smartphone battery to last more than on the other two experimental scenarios, the precision of the system services is also lower in this case. In the activity detection scenario, as the user activity is detected less often, it is more likely that some user movements are not detected. It generates less location updates, resulting on a system performance degradation. On the fall detection system, the probability of a fall not being detected increases as less frequent the acceleration and angular velocity values are checked by the Shimmer module. This is due to the time interval on which a fall starts to be noticed being as small as possible, since a fall is originated by a sudden movement in the order of milliseconds.

The chosen solution to be deployed on the AMBRO mobile gateway was the one evaluated at the first experimental scenario, due to the better commitment between the power consumption (battery of the smartphone and the smart watch) and the overall system performance. It was assured that no important information is lost during the operation of each service available at the AMBRO mobile gateway, while the power used to make those services operate not too prejudicial for the system.

\subsection{Accuracy of the monitoring services}

By assessing the accuracy of the results returned by the monitoring services of the AMBRO mobile gateway it is possible to evaluate if it is conducting his job properly. The location monitoring service accuracy can be obtained by evaluating the operating mode of the mechanisms used to detect the user activity and the GPS coordinates. The activity detection mechanism of the AMBRO mobile gateway was used to detect whether the user was quiet in some place or in movement. If the user stops and not uses his/her smartphone for any purpose, the activity detection mechanism always returns the user is stopped. If the user is walking or driving a car, the activity detection mechanism detects the user is in movement, requesting location updates through the most accurate location provider available at the moment (network, power cells or GPS). The location update mechanism is more or less accurate depending on the location provider that is used to get the GPS coordinates. For example, if the location update mechanism is capable to use a GPS provider to get coordinates, the accuracy can change from 10 up to 30 meters; if the location update mechanism is only capable of using the power cells to get user location, the accuracy can drop to distances in order of 2000 meters. To test the accuracy of the location monitoring service, there were collected 1800 GPS coordinates samples when the user was outdoor and another 1800 GPS coordinates samples when the user was indoor. Figure 7 illustrates the results obtained to evaluate the accuracy of the location monitoring service when the user was indoor and outdoor.

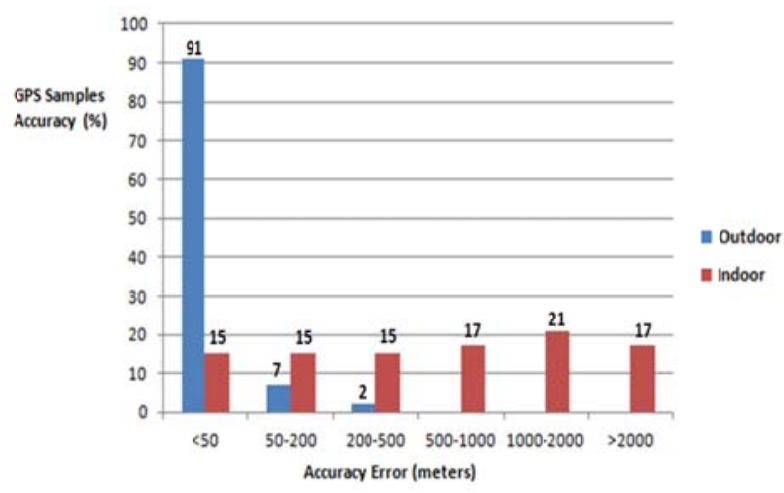

Fig.7. GPS accuracy error of the location monitoring service.

GPS coordinates are more accurate in outdoor locations with $91 \%$ of the gathered samples having accuracy better than 50 meters. In comparison, at indoor locations, only $15 \%$ of the samples have accuracy better than 50 meters. These results were already expected, since at outdoor locations the smartphone is abler to get the location coordinates through the GPS system, while at indoor the coordinates are usually gathered through the Wi-Fi network.

The results returned by the heart rate monitoring service are dependent of the accuracy of the optical heart rate sensor embedded in the smart watch. The accuracy on those types of sensors is good when the person stills quiet and deteriorates when the person is in movement. As the optical heart rate sensor embedded in the smart watch sometimes returns heartbeat values equal to zero, the service cannot be used to detect if a person faints or collapses since it could detect too many false positive values. Instead, the service evaluates the usual heartbeat when the person is still and under movement. Figure 8 illustrates the percentage of non-zero values for scenarios where the person using the smart watch was still, and other scenarios where the person was moving. For each scenario 18000 samples were gathered.

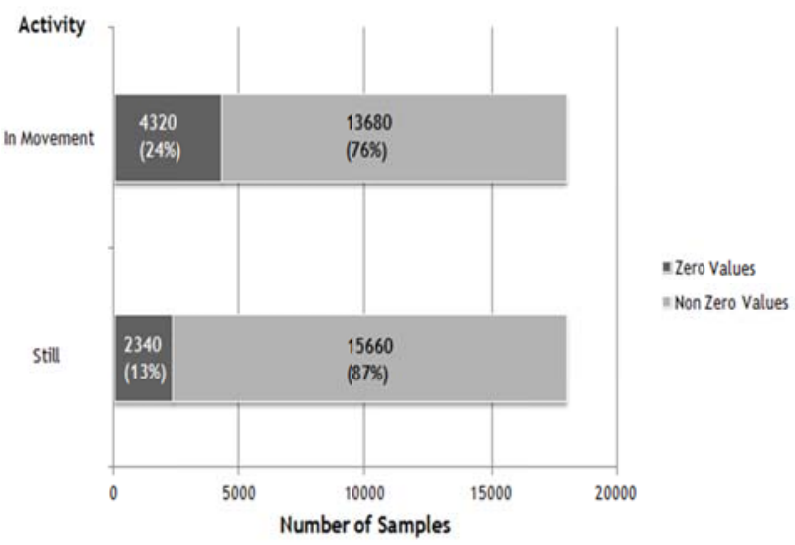

Fig.8. Accuracy of the heart rate monitoring service considering a person quiet and in movement. 
Analyzing the results shown on Figure 8, while the user was stopped the optical heart rate sensor of the Moto 360 returned values different to zero around $87 \%$ of the time. When the user was moving the results only shown valid values about $76 \%$ of the time. This can be related to the displacement of the smart watch in the wrist while the user is moving, as previously expected.

The fall detection service must only detect a fall when a person effectively falls. But sometimes, when a person is performing some daily life activities, the algorithm used by the accelerometer and the gyroscope can fail and detect false positives. Two examples of daily life activities that can generate false positive results are running and jumping, since the thresholds used to detect falls can be sometimes surpassed. To evaluate the accuracy of the fall detection service, it was ascertained the number of falls the Shimmer module detected when its user was performing different daily life activities. Table V shows the results of the experiments performed to evaluate the accuracy of the fall detection service.

Table V. Results of the experiments performed to evaluate the accuracy of the fall detection service.

\begin{tabular}{|c|c|c|c|}
\hline \multirow{2}{*}{$\begin{array}{l}\text { Activities } \\
\text { Performed }\end{array}$} & \multicolumn{3}{|c|}{$\begin{array}{l}\text { Falls detected when performing different daily life } \\
\text { activities }\end{array}$} \\
\hline & $\begin{array}{l}\text { Number of } \\
\text { experiments }\end{array}$ & Falls detected & $\begin{array}{c}\text { Algorithm } \\
\text { accuracy (\%) }\end{array}$ \\
\hline Still & 30 & 0 & $100 \%$ \\
\hline Walk & 30 & 0 & $100 \%$ \\
\hline Sit down & 30 & 0 & $100 \%$ \\
\hline Stand up & 30 & 0 & $100 \%$ \\
\hline Lay down & 30 & 0 & $100 \%$ \\
\hline Jump & 30 & 1 & $96.7 \%$ \\
\hline Run & 30 & 4 & $86.7 \%$ \\
\hline Fall & 30 & 28 & $93.3 \%$ \\
\hline
\end{tabular}

The analysis of the Table $\mathrm{V}$ allows the identification of two situations where falls were detected when they did not really occurred (false positives), i.e. when the user was jumping, and when the user was running. On the 30 experiments performed while the user was jumping, the Shimmer module identified 1 fall incorrectly, originating an accuracy of $96.7 \%$. When the user was running there were detected 4 incorrectly situations, originating an $86.7 \%$ accuracy. For the 30 falls experiments 28 were correctly identified, giving $93.3 \%$ accuracy for the service.

\subsection{Interoperability with other objects}

The AMBRO mobile gateway is able to search other objects in the environment through Bluetooth broadcast messages. This happens when the object search service is used. Searching Bluetooth objects in the environment is a process that can cause a high battery drain and should not be performed frequently. Despite that, using sensors from different vendors, like the embedded heart rate sensor from the Motorola smart watch, Shimmer sensors, and the embedded sensors from the smartphone that acts as gateway itself, the AMBRO mobile gateway uses a very important concept in IoT environments: the interoperability between objects [4][43]. One way to improve the overall performance of the system could be the use of BLE sensors instead of Bluetooth sensors, since BLE allows sensors to announce the services provided by them to other objects through a client-server model.

\section{Conclusion and Future Work}

AMBRO is an IPA platform able to be included in ubiquitous communication scenarios. It is supposed to help their owners on many of their daily tasks, acting on many of those situations in an autonomous way. The architecture of the IPA platform defines the existence of several user interfaces that AMBRO users can use, such as a cloud infrastructure that allows the processing of many mechanisms of the AMBRO platform and an external environment comprising several intelligent objects that AMBRO can interact with. In this last architecture layer, gateways are needed to forward traffic between objects. Static gateways can be used for static environments (smart home, for example). Mobile gateways are used on mobile environments where objects are moving (mobile health scenarios), like the scenario explored in this paper. This scenario comprised the use of a Shimmer sensor module for falls detection, an optical heart rate sensor embedded on a smart watch to detect the user heart rate, and a GPS sensor included on a smartphone to detect the user location. All the information gathered by those sensors is important for caretakers using the AMBRO personal assistant, since it allows them to check the health status of persons under their care anytime and anywhere.

The construction of the AMBRO mobile gateway application allowed the creation of a ubiquitous communication scenario where heterogeneous devices (the Samsung smartphone, the Motorola smart watch and the Shimmer sensor device) can communicate smoothly. Besides that, it also helped to prove that AMBRO personal assistant can be used on different types of environments. Nevertheless, some work can be performed in the future in order to improve the performance of the mobile gateway. One possible improvement would be the use of sensors communicating through Bluetooth Low Energy, since it would allow those sensors to announce the services offered by them to the gateway, diminishing at the same time the power consumption needed on the communication process. Other improvements that can be associated are the following: extend the mobile gateway application to other mobile operating systems, such as iOS or Windows phone; development of new communication mechanisms 
using protocols that consume less energy, such as the constrained application protocol (CoAP); and the inclusion of more sensors on the BSN, increasing the number of physiological parameters to be monitored.

\section{Acknowledgments}

This work has been partially supported by TIMWE Investigação $e$ Desenvolvimento, through the AMBRO project, co-funded by COMPETE/QREN/EU, in the context of the Portuguese Sistema de Incentivos à I\&DT (project 33779), by the National plan of Science, Technology and Innovation (MAARIFAH), King Abdulaziz City for Science and Technology, Kingdom of Saudi Arabia, Award Number (12-INF2817-02), by Government of Russian Federation, Grant 074-U01, by Instituto de Telecomunicações, Next Generation Networks and Applications Group (NetGNA), Covilhã Delegation, by National Funding from the FCT - Fundação para a Ciência e a Tecnologia through the UID/EEA/500008/2013 Project.

\section{References}

[1] G. Czibula, A.-M. Guran, I. G. Czibula, and G. S. Cojocar, "IPA An intelligent personal assistant agent for task performance support" in 2009 IEEE 5th International Conference on Intelligent Computer Communication and Processing, Cluj-Napoca, Romania, August 27-29, 2009, pp. 31-34.

[2] S. Pais, J. Casal, R. Ponciano, and S. Lourenço, "Unsupervised Assistive and Adaptive Intelligent Agent in Smart Environment" in Conference: ICIES 2015: International Conference on Intelligent Environments and Systems, Paris, France, January 23-24, 2015.

[3] L. Atzori, A. Iera, and G. Morabito, "The Internet of Things: A survey", in Comput. Networks, vol. 54, no. 15, pp. 2787-2805, October 2010 .

[4] L. Tan and N. Wang, "Future internet: The Internet of Things" in 2010 3rd International Conference on Advanced Computer Theory and Engineering(ICACTE), Chengdu, China, August 20-22, 2010, pp. 376-380.

[5] P-V. Mekikis, E. Kartsakli, A. Antonopoulos, A. S. Lalos, L. Alonso, C. Verikoukis, "Information Exchange in Randomly Deployed Dense WSNs with Wireless Energy Harvesting Capabilities", in IEEE Transactions on Wireless Communications, 2016.

[6] P. Jäppinen, R. Guarneri and L. M. Correia, "An Applications Perspective into the Future Internet", in Journal of Network and Computer Applications, Elsevier, vol. 36, no. 1, pp. 249-254, January 2013.

[7] K. Bing, L. Fu, Y. Zhuo, and L. Yanlei, "Design of an Internet of Things-based smart home system" in 2011 2nd Int. Conf. Intell. Control Inf. Process., Harbin, China, July 25-28, 2011, pp. 921924

[8] M. Soliman, T. Abiodun, T. Hamouda, J. Zhou, and C.-H. Lung, "Smart Home: Integrating Internet of Things with Web Services and Cloud Computing" in 2013 IEEE 5th International Conference on Cloud Computing Technology and Science (CloudCom), Bristol, United Kingdom, December 2-5, 2013, pp. 317-320.

[9] A. Zanella, N. Bui, A. Castellani, L. Vangelista, and M. Zorzi, "Internet of Things for Smart Cities", in IEEE Internet Things J., vol. 1, no. 1, pp. 22-32, February 2014

[10] Z. Bi, L. Da Xu, and C. Wang, "Internet of Things for Enterprise Systems of Modern Manufacturing", in IEEE Trans. Ind. Informatics, vol. 10, no. 2, pp. 1537-1546, May 2014.
[11] D. Singh, U. S. Tiwary, and W.-Y. Chung, "IP-based ubiquitous healthcare system" in 2008 International Conference on Control, Automation and Systems (ICCAS), Seoul, South Korea, October 14-17, 2008, pp. 131-136.

[12] M. C. Domingo, "An Overview of the Internet of Things for People with Disabilities", in Journal of Network and Computer Applications, Elsevier, vol. 35, no. 2, pp. 584-596, March 2012.

[13] W. Liu and Z. Gao, "Study on IOT based Architecture of Logistics Service Supply", in Int. J. Grid Distrib. Comput., vol. 7, no. 1, pp. 169-178, February 2014.

[14] A. Menon, R. Sinha, D. Ediga, I. Technology, and O. A. Road, "Implementation of Internet of Things in Bus Transport System of Singapore", in Asian J. Eng. Res., vol.1, no.4, pp.8-17, JulySeptember 2013.

[15] J. Serra, D. Pubill, A. Antonopoulos and C. Verikoukis, "Smart HVAC Control in IoT: Energy Consumption Minimization with User Comfort Constraints", in The Scientific Worl Journal, Hindawi Publishing Corporation, vol. 2014, pp. 1-11, June 2014.

[16] B. M. C. Silva, J. J. P. C. Rodrigues, I. de la Torre Díez, M. López-Coronado, and K. Saleem, "Mobile-health: A review of current state in 2015", in J. Biomed. Inform., vol. 56, pp. 265-272, August 2015.

[17] J. J. P. C. Rodrigues, O. R. E. Pereira, and P. A. C. S. Neves, "Biofeedback Data Visualization for Body Sensor Networks", in Journal of Network and Computer Applications, Elsevier, Vol. 34, No. 1, pp. 151-158, January 2011.

[18] "Apple Siri Webpage." [Online]. Available: https://www.apple.com/ios/siri/. Accessed 24 July 2015.

[19] "Google Now Webpage." [Online]. Available: http://www.google.com/landing/now/. Accessed 24 July 2015.

[20] "Samsung S Voice Webpage." [Online]. Available: http://www.samsung.com/global/galaxys $3 /$ svoice.html. Accessed 24 July 2015

[21] "Microsoft Cortana Webpage." [Online]. Available: http://www.windowsphone.com/en-us/how-to/wp8/cortana/meetcortana. Accessed 24 July 2015.

[22] B. M. Silva, I. M. Lopes, M. B. Marques, J. J. P. C. Rodrigues, and M. L. P. Jr, "A Mobile Health Application for Outpatients Medication Management" in IEEE International Conference on Communications, Budapest, Hungary, June 9-13, 2013, pp. 43894393.

[23] J. J. P. C. Rodrigues, I. M. C. Lopes, B. M. C. Silva, and I. D. La Torre, "A new mobile ubiquitous computing application to control obesity: SapoFit.", in Inform. Health Soc. Care, vol. 38, no. 1, pp. 37-53, January 2013

[24] A. Komninos and S. Stamou, "HealthPal : An Intelligent Personal Medical Assistant for Supporting the Self-Monitoring of Healthcare in the Ageing Society" in 4th International Workshop on Ubiquitous Computing for Pervasive Healthcare Applications (UbiComp 2006), California, USA, September 17-21, 2006.

[25] E. T. Horta, I. C. Lopes, J. J. P. C. Rodrigues, and S. Misra, "Real Time Falls Prevention and Detection with Biofeedback Monitoring Solution for Mobile Environments" in IEEE 15th Int. Conf. eHealth Networking, Appl. Serv. (IEEE HEALTHCOM 2013), Lisbon, Portugal, October 9-12, 2013, pp. 594-600.

[26] A. Schrader, D. Carlson, and P. Rothenpieler, "SmartAssist Wireless Sensor Networks for Unobtrusive Health Monitoring" in 5th Workshop on Behaviour Monitoring and Interpretation (BMII'10), Karlsruhe, Germany, September 21, 2010, pp. 84-89.

[27] G. Virone, A. Wood, L. Selavo, Q. Cao, L. Fang, T. Doan, Z. He, R. Stoleru, S. Lin, and J. A. Stankovic, "An Advanced Wireless 
Sensor Network for Health Monitoring" in Transdisciplinary Conference on Distributed Diagnosis and Home Healthcare (D2H2), Arlington, VA, USA, April 2-4, 2006, pp. 2-5.

[28] D. Malan, T. Fulford-Jones, M. Welsh, and S. Moulton, "CodeBlue: An Ad Hoc Sensor Network Infrastructure for Emergency Medical Care" in International Workshop on Wearable and Implantable Body Sensor Networks, London, United Kingdom, April 6-7, 2004

[29] S. Tennina, M. Di Renzo, E. Kartsakli, F. Graziosi, Aris S. Lalos, A. Antonopoulos, P.V. Mekikis and L. Alonso, "WSN4QoL: A WSN-Oriented Healthcare System Architecture" in International Journal of Distributed Sensor Networks, vol. 2014, pp. 1-16, 2014.

[30] E. Kartsakli, A. Lalos, A. Antonopoulos, S. Tennina, M. Di Renzo, L. Alonso, C. Verikoukis, "A Survey on M2M Systems for mHealth: A Wireless Communications Perspective" in Sensors, vol. 14, no.3, pp. 18009-18052, September 2014.

[31] A. Lalos, E. Kartsakli, A. Antonopoulos, S. Tenina, M. Di Renzo, L. Alonso and C. Verikoukis, "RLNC-Aided Cooperative Compressed Sensing for Energy Efficient Vital Signal Telemonitoring"in IEEE Transactions on Wireless Sensor Networks, vol. 14, no.7, pp. 3685-3699, July 2015.

[32] E. Kartsakli, A. Antonopoulos, L. Alonso and C. Verikoukis, "A Cloud-Assisted Random Linear Network Coding Medium Access Control Protocol for Healthcare Applications" in Sensors, vol. 14, no.3, pp. 4806-4830, March 2014.

[33] P. Desai, A. Sheth, and P. Anantharam, "Semantic Gateway as a Service architecture for IoT Interoperability" in IEEE 4th International Conference on Mobile Services, New York, NY, USA, June 27-July 2, 2015.

[34] J. Granados, A.-M. Rahmani, P. Nikander, P. Liljeberg, and H. Tenhunen, "Towards Energy-Efficient HealthCare: an Internet-ofThings Architecture Using Intelligent Gateways" in Proceedings of the 4th International Conference on Wireless Mobile Communication and Healthcare, Athens, Greece, November 3-5, 2014, pp.279-282.

[35] C.-H. Wang, "UPHSM: Ubiquitous personal health surveillance and management system via WSN agent on open source smartphone" in 2011 IEEE 13th International Conference on eHealth Networking, Applications and Services (Healthcom 2011), Columbia, MO, USA, June 13-15, 2011, pp. 60-63.

[36] P. Santos, L. Varandas, T. Alves, C. Romeiro, J. Casal, S. Lourenço, and J. Santos, "A Pervasive System Architecture for Smart Environments in Internet of Things Context" in ICMI
2015 : XVII International Conference on Multimodal Interaction, London, United Kingdom, January 19-20, 2015.

[37] "SHIMMER Sensing platform Webpage" [Online]. Available: http://www.shimmersensing.com/. Accessed 30 May 2015.

[38] "Activity Recognition API Webpage." [Online]. Available: https://developers.google.com/android/reference/com/google/andro $\mathrm{id} /$ gms/location/ActivityRecognitionApi. Accessed 2 July 2015.

[39] S. Vanjire, U. Kanchan, G. Shitole, and P. Patil, "Location Based Services on Smart Phone through the Android Application" in Int J. Adv. Res. Comput. Commun. Eng., vol. 3, no. 1, pp. 4982-4987, January 2014.

[40] A. Chavan, S. Kamble, A. Bhagyshree, R. Sonawane, and R. C. Gadri, "Land Vehicle Tracking System Using Java on Android Platform", in Comput. Eng. Intell. Syst., vol. 3, no. 5, pp. 88-94, June 2012

[41] A. Al-Mazloum, E. Omer, and M. F. A. Abdullah, "GPS and SMSBased Child Tracking System Using Smart Phone", in Int. $J$ Electr. Comput. Electron. Commun. Eng., vol. 7, no. 2, pp. 171$174,2013$.

[42] R. Kinage, J. Kumari, P. Zalke, and M. Kulkarni, "Mobile Tracking Application", in Int. J. Innov. Res. Sci. Eng. Technol., vol. 2, no. 3, pp. 617-623, March 2013

[43] A. J. Jara, L. Ladid, and A. Skarmeta, "The Internet of Everything through IPv6 : An Analysis of Challenges, Solutions and Opportunities", in J. Wirel. Mob. Networks, Ubiquitous Comput. Dependable Appl., vol. 4, no. 3, pp. 97-118, September 2013.

[44] "Android Wear Wearable Message API." [Online]. Available: https://developer.android.com/training/wearables/datalayer/messages.html. Accessed 7 July 2015.

[45] Q. T. Huynh, U. D. Nguyen, S. V Tran, A. Nabili, and B. Q. Tran, "Fall Detection System Using Combination Accelerometer and Gyroscope" in Proceedings of the Second International Confernece on Advances in Electronic Devices and Circuits (EDC 2013), Kuala Lumpur, Malaysia, May 4-5, 2013, pp. 52-56.

[46] S. Hwang, M. Ryu, Y. Yang, and N. Lee, "Fall Detection with Three-Axis Accelerometer and Magnetometer in a Smartphone" in International Conference on Computer Science and Technology, CST 2012, Jeju, South Korea, June 25-27, 2012, pp. 65-70.

[47] E. Lee and C. Kim, "An Intelligent Green Service in Internet of Things”, in J. Converg., vol. 5, no. 3, pp. 4-8, September 2014. 


\section{A IoT-based Mobile Gateway for Intelligent Personal Assistants on Mobile Health Environments}

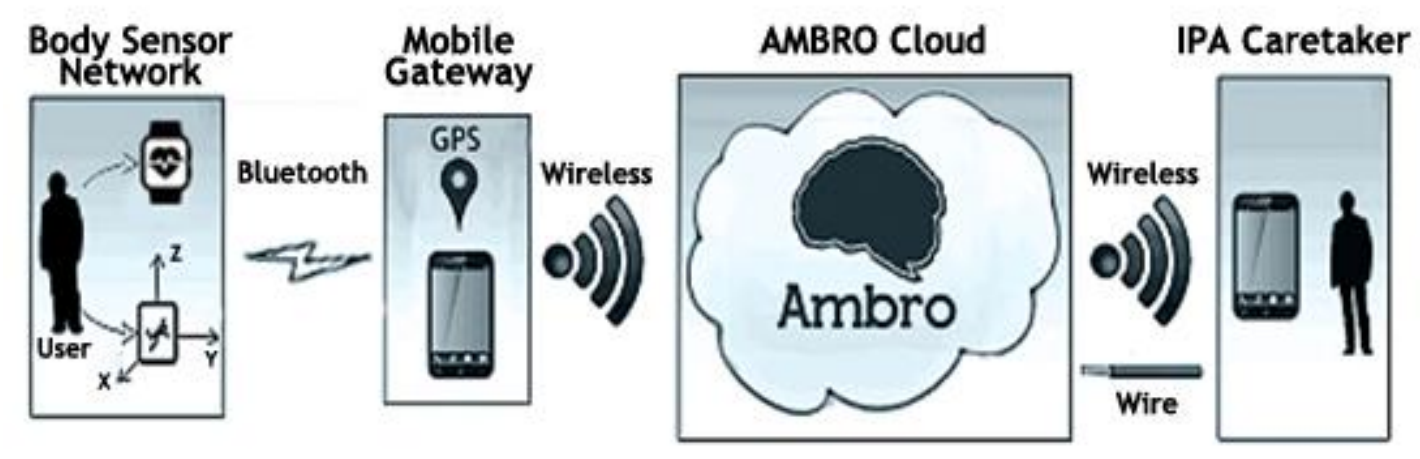

Intelligent Personal Assistants (IPAs) are software agents used to help users to fulfill several daily actions. Generally, they learn about their user behavior and routines by interacting directly with them. If introduced on ubiquitous communication scenarios, IPAs can interact with other smart objects and improve the knowledge and awareness about their users. One of the potential enablers for ubiquitous communication scenarios is the Internet of Things (loT) paradigm where machines with decision support systems interact and communicate among them. Then, this paper proposes a novel loT-based mobile gateway solution for mobile health (m-Health) scenarios. This gateway autonomously collects information about the user/patient location, heart rate, and possible fall detection. Moreover, it forwards the collected information to a caretaker IPA, in real time, which will manage a set of actions and alarms appropriately. 